\title{
CERTAIN RESULTS ON $N(k)$-CONTACT METRIC MANIFOLDS
}

\author{
UDAY CHAND DE
}

\begin{abstract}
In the present paper we study contact metric manifolds whose characteristic vector field $\xi$ belonging to the $k$-nullity distribution. First we consider concircularly pseudosymmetric $N(k)$-contact metric manifolds of dimension $(2 n+1)$. Beside these, we consider Ricci solitons and gradient Ricci solitons on three dimensional $N(k)$-contact metric manifolds. As a consequence we obtain several results. Finally, an example is given.
\end{abstract}

\section{Introduction}

In 1982, R. S. Hamilton [31] introduced the notion of Ricci flow to find a canonical metric on a smooth manifold. The Ricci flow is an evolution equation for metrics on a Riemannian manifold defined as follows:

$$
\frac{\partial}{\partial_{t}} g_{i j}=-2 R_{i j}
$$

A Ricci soliton is a generalization of an Einstein metric. We recall the notion of Ricci soliton according to [14]. On the manifold $M$, a Ricci soliton is a triple $(g, V, \lambda)$ with $g$, a Riemannian metric, $V$ a vector field, called potential vector field and $\lambda$ a real scalar such that

$$
\$_{V} g+2 S+2 \lambda g=0 \text {, }
$$

where $\$$ is the Lie derivative. Ricci solitons are special solutions of the Ricci flow equation (1.1) of the form $g_{i j}=\sigma(t) \psi_{t}^{*} g_{i j}$ with the initial condition $g_{i j}(0)=g_{i j}$, where $\psi_{t}$ are diffeomorphism of $M$ and $\sigma(t)$ is the scaling function.

Metrics satisfying (1.2) are interesting and useful in physics and are often referred as quasi-Einstein ([16],[17]). Compact Ricci solitons are the fixed points of the Ricci flow $\frac{\partial}{\partial t} g=$ $-2 S$ projected from the space of metrics onto its quotient modulo diffeomorphisms and scalings, and often arise blow-up limits for the Ricci flow on compact manifolds. Theoretical

Received June 23, 2017, accpted April 27, 2018.

2010 Mathematics Subject Classification. 53C15, 53C25.

Key words and phrases. $N(k)$-contact metric manifolds, concircularly pseudosymmetric, Ricci soliton, Gradient Ricci soliton, Einstein manifolds. 
physicists have also been looking into the equation of Ricci soliton in relation with string theory. The initial contribution in this direction is due to Friedan [30] who discusses some aspects of it.

The Ricci soliton is said to be shrinking, steady and expanding according as $\lambda$ is negative, zero and positive. If the vector field $V$ is the gradient of a potential function $-f$, then $g$ is called a gradient Ricci soliton and equation (1.2) takes the form

$$
\nabla \nabla f=S+\lambda g
$$

where $\nabla$ denotes the Riemannian connection.

A Ricci soliton on a compact manifold has constant curvature in dimension 2 (Hamilton [31]), and also in dimension 3 (Ivey [33]). For details we refer to Chow and Knoff [22]. We also recall the following significant result of Perelman [38]: A Ricci soliton on a compact manifold is a gradient Ricci soliton.

Since the last two decades, the geometry of Ricci solitons has been the focus of attention of many mathematicians. In particular, it has become more important after Grigory Perelman solved the Poincare conjecture. An Einstein manifold is a trivial example of a gradient Ricci soliton with constant potential function and therefore it is called a trivial Ricci soliton. There exist many non-trivial examples of Ricci solitons which is compact as well as non-compact ([22], [33], [34]).

There are two aspects of the study of Ricci solitons, one looking at the influence on the topology by the Ricci soliton structure of the Riemannian manifold ([27],[46]) and the other looking at its influence on its geometry ([28], [29]).

On the other hand, the roots of contact geometry lie in differential equations as in 1872 Sophus Lie introduced the notion of contact transformation as a geometric tool to study systems of differential equations. This subject has manifold connections with the other fields of pure mathematics and substantial applications in applied areas such as mechanics, optic, phase space of dynamical system, thermodynamics and control theory.

Recently in [14] C. Calin and M. Crasmareanu have studied Ricci solitons in $f$-Kenmotsu manifolds. Also in [1] Bejan et al. studied Ricci solitons in manifolds with quasi-constant curvature. Also Ghosh et al. [32] studied gradient Ricci soliton in $(k, \mu)$-contact metric manifolds. Ricci soliton have been studied by several authors such as ([18], [19], [20], [21]) and many others.

Let $(M, g)$ be a Riemannian manifold and let $\nabla$ be the Levi-Civita connection of $(M, g)$. A Riemannian manifold is called locally symmetric [7] if $\nabla R=0$, where $R$ is the Riemannian curvature tensor of $(M, g)$. A Riemannian manifold $M$ is called semisymmetric if

$$
R \cdot R=0
$$


holds, where $R$ denotes the curvature tensor of the manifold. It is well known that the class of semisymmetric manifolds includes the set of locally symmetric manifolds $(\nabla R=0)$ as a proper subset. Semisymmetric Riemannian manifolds were first studied by E. Cartan, A. Lichnerowich, R. S. Couty and N. S. Sinjukov. A fundamental study on Riemannian semisymmetric manifolds was made by Z. I. Szabó [39], E. Boeckx et al [5] and O. Kowalski [13]. A Riemannian manifold $M$ is said to be Ricci semisymmetric if on $M$ we have

$$
R \cdot S=0
$$

where $S$ is the Ricci tensor.

The class of Ricci-semisymmetric manifolds includes the set of Ricci symmetric manifolds $(\nabla S=0)$ as a proper subset. Ricci-semisymmetric manifolds were investigated by several authors. We define the subsets $U_{R}, U_{S}$ of a Riemannian manifold $M$ by $U_{R}=\{x \in M$ : $R-\frac{\kappa}{(n-1) n} G \neq 0 \quad$ at $\left.\quad x\right\}$ and $U_{S}=\left\{x \in M: S-\frac{\kappa}{n} g \neq 0 \quad\right.$ at $\left.\quad x\right\}$ respectively, where $G(X, Y) Z=$ $g(Y, Z) X-g(X, Z) Y$ and $\kappa$ is the scalar curvature. Evidently we have $U_{S} \subset U_{R}$. A Riemannian manifold is said to be pseudo-symmetric [41] if at every point of $M$ the tensor $R \cdot R$ and $Q(g, R)$ are linearly dependent. This is equivalent to

$$
R \cdot R=f_{R} Q(g, R)
$$

on $U_{R}$, where $f_{R}$ is some function on $U_{R}$. Clearly, every semi-symmetric manifold is pseudosymmetric but the converse is not true [41].

A Riemannian manifold $M$ is said to Ricci pseudo-symmetric if $R \cdot S$ and $Q(g, S)$ on $M$ are linearly dependent. This is equivalent to

$$
R \cdot S=f_{S} Q(g, S)
$$

holds on $U_{S}$, where $f_{S}$ is a function defined on $U_{S}$.

In [4] Blair et al. studied $N(k)$-contact metric manifolds satisfying the curvature conditions $\tilde{Z} \cdot \tilde{Z}=0, \tilde{Z} \cdot R=0$ and $R \cdot \tilde{Z}=0$, where $\tilde{Z}$ is the concircular curvature tensor defined ([43], [44])by

$$
\tilde{Z}(X, Y) W=R(X, Y) W-\frac{r}{2 n(2 n+1)}[g(Y, W) X-g(X, W) Y],
$$

where $X, Y, W \in T M$ and $r$ is the scalar curvature. Recently, De et al. [8] studied $N(k)$-contact metric manifolds satisfying the curvature conditions $P \cdot R=0, P \cdot S=0$ and $P \cdot P=0$.

The purpose of this paper is to generalize a result of Blair [4] in a $(2 n+1)$-dimensional $N(k)$-contact metric manifold. Further we characterize three dimensional $N(k)$-contact metric manifold admitting Ricci soliton and gradient Ricci soliton.

The present paper is organized as follows: 
After preliminaries in section 3, we consider concircularly pseudosymmetric $N(k)$-contact metric manifolds of dimension $(2 n+1)$. In Section 4 we study Ricci solitons on three dimensional $N(k)$-contact metric manifolds. Section 5 deals with gradient Ricci solitons on three dimensional $N(k)$-contact metric manifolds. Finally, an example is given.

\section{Preliminaries}

A contact manifold is by definition an odd dimensional manifold $M^{2 n+1}$ equipped with a global 1-form satisfying $\eta \wedge(d \eta)^{n} \neq 0$ everywhere. It is well-known that there exists a unique vector field $\xi$, the characteristic vector field for which $\eta(\xi)=1$ and $i_{\xi} d \eta=0$. Further, one can find an associated Riemannian metric $g$ and a vector field $\phi$ of type $(1,1)$ such that

$$
\eta(X)=g(X, \xi), d \eta(X, Y)=g(X, \phi Y), \phi^{2} X=-X+\eta(X) \xi
$$

where $X$ and $Y$ are vector fields on $M$. From (2.1) it follows that

$$
\phi \xi=0, \eta \circ \phi=0, g(\phi X, \phi Y)=g(X, Y)-\eta(X) \eta(Y)
$$

The manifold $M^{2 n+1}$ together with the structure tensor $(\eta, \xi, \phi, g)$ is called a contact metric manifold ([2], [3]).

Given a contact metric manifold $(M, \eta, \xi, \phi, g)$, we define a symmetric $(1,1)$-tensor field $h$ as $h=\frac{1}{2} L_{\xi} \phi$, where $L_{\xi} \phi$ denotes Lie differentiation in the direction of $\xi$. We have the following identities:

$$
\begin{aligned}
& h \xi=0, \quad h \phi+\phi h=0, \\
& \nabla_{X} \xi=-\phi X-\phi h X, \\
& \nabla_{\xi} \phi=0, \\
& R(\xi, X) \xi-\phi R(\xi, \phi X) \xi=2\left(h^{2}+\phi^{2}\right) X, \\
& \left(\nabla_{\xi} h\right) X=\phi X-h^{2} \phi X+\phi R(\xi, X) \xi, \\
& S(\xi, \xi)=2 n-\operatorname{tr} h^{2}, \\
& R(X, Y) \xi=-\left(\nabla_{X} \phi\right) Y+\left(\nabla_{Y} \phi\right) X-\left(\nabla_{X} \phi h\right) Y+\left(\nabla_{Y} \phi h\right) X .
\end{aligned}
$$

Here, $\nabla$ is the Levi-Civita connection and $R$ the Riemannian curvature tensor of $(M, g)$ with the sign convention

$$
R(X, Y) Z=\nabla_{X} \nabla_{Y} Z-\nabla_{Y} \nabla_{X} Z-\nabla_{[X, Y]} Z
$$

for vector fields $X, Y, Z$ on $M$. The tensor $l=R(\cdot, \xi) \xi$ is the Jacobi operator with respect to the characteristic field $\xi$. 
If the characteristic vector field $\xi$ is a Killing vector field, the contact metric manifold $(M, \eta, \xi, \phi, g)$ is called $K$-contact manifold. This is the case if and only if $h=0$. Finally, if the Riemann curvature tensor satisfies

$$
R(X, Y) \xi=\eta(Y) X-\eta(X) Y
$$

or, equivalently, if

$$
\left(\nabla_{X} \phi\right) Y=g(X, Y) \xi-\eta(Y) X
$$

holds, then the manifold is Sasakian. We note that a Sasakian manifold is always $K$-contact, but the converse only holds in dimension three.

The $k$-nullity distribution $N(k)$ of a Riemannian manifold is defined by [45]

$$
N(k): p \longrightarrow N_{p}(k)=\left\{Z \in T_{p} M: R(X, Y) Z=k[g(Y, Z) X-g(X, Z) Y]\right\},
$$

$k$ being a real number. If the characteristic vector field $\xi \in N(k)$, then we call a contact metric manifold as $N(k)$-contact metric manifold [45]. If $k=1$, then the manifold is Sasakian and if $k=0$, then the manifold is locally isometric to the product $E^{n+1}(0) \times S^{n}(4)$ for $n>1$ and flat for $n=1$ [2].

However, for a $N(k)$-contact metric manifold $M$ of dimension $(2 n+1)$, we have [4]

$$
\left(\nabla_{X} \phi\right) Y=g(X+h X, Y) \xi-\eta(Y)(X+h X),
$$

where $h=\frac{1}{2} \$_{\xi} \phi$,

$$
\begin{aligned}
h^{2}= & (k-1) \phi^{2} \\
R(X, Y) \xi= & k[\eta(Y) X-\eta(X) Y], \\
S(X, Y)= & 2(n-1) g(X, Y)+2(n-1) g(h X, Y) \\
& +[2 n k-2(n-1)] \eta(X) \eta(Y), \quad n \geq 1 . \\
S(Y, \xi)= & 2 n k \eta(X), \\
\left(\nabla_{X} \eta\right)(Y)= & g(X+h X, \phi Y), \\
\left(\nabla_{X} h\right)(Y)= & \{(1-k) g(X, \phi Y)+g(X, h \phi Y)\} \xi+\eta(Y)[h(\phi X+\phi h X)],
\end{aligned}
$$

for any vector fields $X, Y, Z$ where $R$ is the Riemannian curvature tensor and $S$ is the Ricci tensor. $N(k)$-contact metric manifolds have been studied by several authors such as ([9], [10], [11], [25], [35], [36], [37]) and many others.

The curvature tensor of a 3-dimensional Riemannain manifold is given by

$$
\begin{aligned}
R(X, Y) Z= & {[S(Y, Z) X-S(X, Z) Y+g(Y, Z) Q X-g(X, Z) Q Y] } \\
& -\frac{r}{2}[g(Y, Z) X-g(X, Z) Y],
\end{aligned}
$$


where $S$ and $r$ are the Ricci tensor and scalar curvature respectively and $Q$ is the Ricci operator defined by $g(Q X, Y)=S(X, Y)$.

In [3] Blair et al proved that in a three dimensional contact metric manifold with $\xi$ belonging to the $k$-nullity distribution, the following conditions hold:

$$
\begin{aligned}
Q X & =\left(\frac{r}{2}-k\right) X+\left(3 k-\frac{r}{2}\right) \eta(X) \xi, \\
S(X, Y) & =\left(\frac{r}{2}-k\right) g(X, Y)+\left(3 k-\frac{r}{2}\right) \eta(X) \eta(Y), \\
Q \phi & =\phi Q, \\
\nabla_{X} \xi & =-(1+\alpha) \phi X,
\end{aligned}
$$

where $\alpha= \pm \sqrt{1-k}$. Contact metric manifolds have also been studied by several authors such as ([3], [23], [24], [42]) and many others.

We also recall the notion of $D$-homothetic deformation. For a given contact metric structure $(\phi, \xi, \eta, g)$, this is the structure defined by

$$
\bar{\eta}=a \eta, \quad \bar{\xi}=\frac{1}{a} \xi, \quad \bar{\phi}=\phi, \quad \bar{g}=a g+a(a-1) \eta \otimes \eta,
$$

where $a$ is a positive constant. While such a change preserves the state of being contact metric, $K$-contact, Sasakian or strongly pseudo-convex $C R$, it detroies a condition like $R(X, Y) \xi=$ 0 or, $R(X, Y) \xi=k\{\eta(Y) X-\eta(X) Y\}$.

However the form of the $(k, \mu)$-nullity condition is preserved under a $D$-homothetic deformation with

$$
\bar{k}=\frac{k+a^{2}-1}{a^{2}}, \quad \bar{\mu}=\frac{\mu+2 a-2}{a}
$$

Given a non-Sasakian $(k, \mu)$-contact manifold $M$, Boeckx [6] introduced an invariant

$$
I_{M}=\frac{1-\frac{\mu}{2}}{\sqrt{1-k}}
$$

and showed that for two non-Sasakian $(k, \mu)$-manifolds $\left(M_{i}, \phi_{i}, \xi_{i}, \eta_{i}, g_{i}\right), i=1,2$, we have $I_{M_{1}}=I_{M_{2}}$ if and only if up to a $D$-homothetic deformation, the two manifolds are locally isometric as contact metric manifolds.

Thus we see that from all non-Sasakian $(k, \mu)$-manifolds locally as soon as we have for every odd dimension $(2 n+1)$ and for every possible value of the invariant $I$, one $(k, \mu)$ manifold $M$ can be obtained with $I_{M}=I$. For $I>-1$ such examples may be found from the standard contact metric structure on the tangent sphere bundle of a manifold of constant curvature $c$ where we have $I=\frac{1+c}{|1-c|}$. Boeckx also gives a Lie algebra construction for any odd dimension and value of $I \leq-1$. 
Example 2.1. Using this invariant, Blair et al. [4] constructed an example of a ( $2 n+1)$-dimensional $N\left(1-\frac{1}{n}\right)$-contact metric manifold, $n>1$. The example is given in the following:

Since the Boeckx invariant for a $\left(1-\frac{1}{n}, 0\right)$-manifold is $\sqrt{n}>-1$, we consider the tangent sphere bundle of an $(n+1)$-dimensional manifold of constant curvature $c$ so chosen that the resulting $D$-homothetic deformation will be a $\left(1-\frac{1}{n}, 0\right)$-manifold. That is, for $k=c(2-c)$ and $\mu=-2 c$ we solve

$$
1-\frac{1}{n}=\frac{k+a^{2}-1}{a^{2}}, \quad 0=\frac{\mu+2 a-2}{a}
$$

for $a$ and $c$. The result is

$$
c=\frac{\sqrt{n} \pm 1}{n-1}, \quad a=1+c
$$

and taking $c$ and $a$ to be these values we obtain $N\left(1-\frac{1}{n}\right)$-contact metric manifold.

The above example will be used in the next section.

\section{Concircularly pseudosymmetric $N(k)$-contact metric manifolds}

A Riemannian manifold is said to be concircularly pseudosymmetric [41] if at every point of the manifold the following relation holds

$$
(R(X, Y) \cdot \tilde{Z})(U, V) W=L_{\tilde{Z}}((X \wedge Y) \cdot \tilde{Z})(U, V) W
$$

for any vector fields $X, Y, U, V, W \in T M$, where $L_{\tilde{Z}}$ is some function of $M$. The endomorphism $X \wedge Y$ is defined by

$$
(X \wedge Y) Z=g(Y, Z) X-g(X, Z) Y
$$

Now a Riemannian manifold is said to be concircularly pseudosymmetric if it satisfies the condition

$$
(R(X, Y) \cdot \tilde{Z})(U, V) W=L_{\tilde{Z}}((X \wedge Y) \cdot \tilde{Z})(U, V) W,
$$

where $L_{\tilde{Z}}$ is some function on $M$.

Let us suppose that a $N(K)$-contact metric manifold satisfies the condition

$$
(R(X, Y) \cdot \tilde{Z})(U, V) W=L_{\tilde{Z}}((X \wedge Y) \cdot \tilde{Z})(U, V) W .
$$

Putting $Y=W=\xi(3.4)$ we have

$$
(R(X, \xi) \cdot \tilde{Z})(U, V) \xi=L_{\tilde{Z}}((X \wedge \xi) \cdot \tilde{Z})(U, V) \xi
$$

It follows that

$$
R(X, \xi) \tilde{Z}(U, V) \xi-\tilde{Z}(R(X, \xi) U, V) \xi-\tilde{Z}(U, R(X, \xi) V) \xi
$$




$$
\begin{aligned}
& -\tilde{Z}(U, V) R(X, \xi) \xi=L_{\tilde{Z}}[(X \wedge \xi) \tilde{Z}(U, V) \xi \\
& -\tilde{Z}((X \wedge \xi) U, V) \xi-\tilde{Z}(U,(X \wedge \xi) V) \xi \\
& -\tilde{Z}(U, V)(X \wedge \xi) \xi] .
\end{aligned}
$$

In view of (1.6), the concircular curvature tensor of a $(2 n+1)$-dimensional $N(k)$ - contact manifold is

$$
\tilde{Z}(X, Y) W=R(X, Y) W-\frac{r}{2 n(2 n+1)}[g(Y, W) X-g(X, W) Y] .
$$

Now from the above equation with the help of (2.12), we have

$$
\tilde{Z}(U, V) \xi=\left(k-\frac{r}{2 n(2 n+1)}\right)[\eta(V) U-\eta(U) V],
$$

for any vector fields $U, V$.

From the equations (3.7) and (3.8) yields

$$
\begin{aligned}
& R(X, \xi) \tilde{Z}(U, V) \xi=\left(k-\frac{r}{2 n(2 n+1)}\right)[\eta(V) R(X, \xi) U-\eta(U) R(X, \xi) V], \\
& \tilde{Z}(R(X, \xi) U, V) \xi=\left(k-\frac{r}{2 n(2 n+1)}\right)[\eta(V) R(X, \xi) U-g(R(X, \xi) U, \xi) V], \\
& \tilde{Z}(U, R(X, \xi) V) \xi=\left(k-\frac{r}{2 n(2 n+1)}\right)[g(R(X, \xi) V, \xi) V-\eta(U) R(X, \xi) V], \\
& \tilde{Z}(U, V) R(X, \xi) \xi=k\left[\tilde{Z}(U, V) X-\frac{r}{2 n(2 n+1)}(\eta(X) \eta(V) U-\eta(X) \eta(U) V)\right] .
\end{aligned}
$$

In view of (3.9), (3.10), (3.11), (3.12) and substituting $V=\xi$ in (3.6) we infer

$$
\begin{aligned}
k^{2}\{\eta(U) \eta(X) \xi-\eta(X) U\}= & -L_{\tilde{Z}}\left(k-\frac{r}{2 n(2 n+1)}\right)\{-3 \eta(U) X \\
& -3 \eta(X) U+X+3 \eta(U) \eta(X) \xi\} .
\end{aligned}
$$

Putting $U=\xi$ in (3.13) we have

$$
L_{\tilde{Z}}\left(k-\frac{r}{2 n(2 n+1)}\right)=0 .
$$

It follows that either $L_{\tilde{Z}}=0$ or $r=2 n k(2 n+1)$. Thus a concircularly pseudosymmetric $N(k)$ contact metric manifold reduces to concircularly semisymmetric $N(k)$-contact metric manifold or $r=2 n k(2 n+1)$. Moreover the scalar curvature of $N(k)$-contact metric manifold is $r=2 n k+2(n-1) 2 n$. Comparing the values of $r$ we get $k=\left(1-\frac{1}{n}\right)$. Thus in view of the above result we can state the following:

Theorem 3.1. $A(2 n+1)$-dimensional concircularly pseudosymmetric $N(k)$-contact metric manifold is either concircularly semisymmetric or locally isometric to the Example 2.1.

Remark 3.1. In [4] Blair et al. studied concircularly semisymmetric $N(k)$-contact metric manifolds. Therefore the above theorem generalizes the result of [4]. 


\section{Ricci solitons}

In particular, let $V$ be collinear with $\xi$ i.e. $V=b \xi$, where $b$ is a function on the three dimensional $N(k)$-contact metric manifold. Then

$$
\left(\$_{V} g+2 S+2 \lambda g\right)(X, Y)=0
$$

which implies that

$$
g\left(\nabla_{X} b \xi, Y\right)+g\left(\nabla_{Y} b \xi, X\right)+2 S(X, Y)+2 \lambda g(X, Y)=0 .
$$

It follows that

$$
\begin{aligned}
& b g\left(\nabla_{X} \xi, Y\right)+(X b) \eta(Y)+b g\left(\nabla_{Y} \xi, X\right) \\
& +(Y b) \eta(X)+2 S(X, Y)+2 \lambda g(X, Y)=0 .
\end{aligned}
$$

Using (2.21) in (4.1), we obtain

$$
\begin{aligned}
& b g(-(1+\alpha) \phi X, Y)+\operatorname{bg}(X,-(1+\alpha) \phi Y) \\
& +(X b) \eta(Y)+(Y b) \eta(X)+2 S(X, Y)+2 \lambda g(X, Y)=0 .
\end{aligned}
$$

In (4.2) replacing $Y$ by $\xi$ it follows that

$$
X b+(\xi b) \eta(X)+4 k \eta(X)+2 \lambda \eta(X)=0 .
$$

Putting $X=\xi$ in (4.3) we get

$$
\xi b=-(2 k+\lambda) .
$$

Putting this value in (4.3), we obtain

$$
X b-(2 k+\lambda) \eta(X)+4 k \eta(X)+2 \lambda \eta(X)=0,
$$

which implies that

$$
d b=-(2 k+\lambda) \eta .
$$

Applying $d$ on (4.6) we get

$$
0=d^{2} b=-(2 k+\lambda) d \eta .
$$

Since $d \eta \neq 0$ we have

$$
\lambda=-2 k \text {. }
$$

Using (4.8) in (4.6) yields $b$ is a constant. Therefore from (4.2) it follows

$$
S(X, Y)=2 k g(X, Y),
$$

which implies that $M$ is an Einstein manifold. This leads to the following: 
Theorem 4.1. In a three dimensional $N(k)$-contact metric manifold, if the metric $g$ is a Ricci soliton and $V$ is collinear with $\xi$, then $V$ is a constant multiple of $\xi$ and $g$ is an Einstein manifold.

In [3] the authors proved that a 3-dimensional $N(k)$-contact metric manifold is either Sasakian, flat or locally isometric to a left invariant metric on the Lie groups $S U(2)$ or $S L(2, \mathbb{R})$. Thus we are in a position to state the following:

Corollary 4.1. In a three dimensional $N(k)$-contact metric manifold, if the metric $g$ is a Ricci soliton and $V$ is collinear with $\xi$, then $V$ is a constant multiple of $\xi$ and the manifold is either Sasakian, flat or locally isometric to a left invariant metric on the Lie groups $S U(2)$ or $S L(2, \mathbb{R})$.

Now, we recall the following result:

Lemma 4.1 ([15]). If $(g, V)$ is a Ricci soliton of a Riemannian manifold, then we have

$$
\frac{1}{2}\left\|\$_{V} g\right\|^{2}=d r(V)+2 \operatorname{div}(\lambda V-Q V),
$$

where $r$ denotes the scalar curvature of $g$ and $Q$ is the Ricci operator defined by $S(X, Y)=$ $g(Q X, Y)$.

In the study of Ricci solitons there are two special situations regarding the vector field $V$ : $V \in \operatorname{span} \xi$ and $V$ orthogonal to $\xi$. But the second class is too complex to analysis. This is why we consider the only case $V=\xi[14]$.

Let $M$ be a three dimensional $N(k)$-contact metric manifold admitting a Ricci soliton whose potential vector field is the Reeb vector field. Then we have from (pp. 48 of [15])

$$
\frac{1}{2}\left(\$_{\xi} g+S+\lambda g\right)(X, Y)=0
$$

which implies that

$$
\frac{1}{2} g\left(\nabla_{X} \xi, Y\right)+g\left(\nabla_{Y} \xi, X\right)+S(X, Y)+\lambda g(X, Y)=0 .
$$

Using (2.4) in (4.12) gives

$$
\begin{aligned}
& -\frac{1}{2}\{g(\phi X, Y)+g(\phi h X, Y)+g(\phi Y, X)+g(\phi h Y, X)\} \\
& +S(X, Y)+\lambda g(X, Y)=0
\end{aligned}
$$

from which we infer

$$
S(X, Y)-g(\phi h X, Y)+\lambda g(X, Y)=0 .
$$

This implies

$$
Q X=\phi h X-\lambda X
$$


Substituting $X=\xi$ in the above equation we get $Q \xi=\lambda \xi$. Since trace of $\phi h$ vanishes, from (4.15) we have the scalar curvature $r=-3 \lambda$, a constant. Now, we assume that $V=\xi$ in (4.10), then we have $\$_{\xi} g=0$, that is, $\xi$ is a Killing vector field. Therefore the manifold becomes a $K$-contact manifold. For three dimensional case a $K$-contact manifold is a Sasakian manifold [12]. Also, since $\xi$ is a Killing vector field, from (4.11) it follows that the manifold is Einstein. Conversely, if the manifold is Sasaki-Einstein, then (4.11) is valid. This leads to the following:

Theorem 4.2. A three dimensional $N(k)$-contact metric manifold admits a Ricci soliton ( $g, \xi$ ) if and only if the manifold is Sasaki-Einstein.

In [40] Sharma proved the following:

Proposition 4.1. Let $(M, \eta, g)$ be a contact metric mnaifold with $\xi$ an eigenvector of the Ricci tensor, and $g$ be a Ricci soliton. Then the Ricci soliton vector field $V$ is Jacobi along $\xi$ if and only if the Ricci curvature along $\xi$ is constant.

In a three dimensional $N(k)$-contact metric manifold, $S(\xi, \xi)=2 n k=$ constant. Therefore in a three dimensional $N(k)$-contact metric manifold Ricci curvature along $\xi$ is constant. An immediate consequence is the following:

Theorem 4.3. If a three dimensional $N(k)$-contact metric manifold admits a Ricci soliton, then $V$ is a Jacobi vector field along the geodesic vector field $\xi$.

\section{Gradient Ricci solitons}

Let $M$ be a three dimensional $N(k)$-contact metric manifold with $g$ as a gradient Ricci soliton. Then the equation (1.3) can be written as

$$
\nabla_{Y} D f=Q Y+\lambda Y
$$

for all vector fields $Y$ in $M$, where $D$ denotes the gradient operator of $g$. From (5.1) it follows that

$$
R(X, Y) D f=\left(\nabla_{X} Q\right) Y-\left(\nabla_{Y} Q\right) X, \quad X, Y \in T M
$$

Using (2.21) we have

$$
g(R(\xi, X) D f, \xi)=k g((D f-(\xi f) \xi), X)
$$

Also in a three dimensional $N(k)$-contact metric manifold, it follows that

$$
g\left(\left(\nabla_{\xi} Q\right) Y-\left(\nabla_{Y} Q\right) \xi, \xi\right)=0
$$


Assuming that $k \neq 0$. Therefore from (5.2), (5.3) and (5.4) we get

$$
k(D f-(\xi f) \xi)=0,
$$

or,

$$
D f=(\xi f) \xi
$$

Using (5.5) in (5.1) we obtain

$$
S(X, Y)+\lambda g(X, Y)=-(1+\alpha)(\xi f) g(\phi Y, X)+Y(\xi f) \eta(X) .
$$

Putting $X=\xi$ in (5.6) we get

$$
Y(\xi f)=(2 k+\lambda) \eta(Y)
$$

From (5.6) and (5.7) we get

$$
S(X, Y)+\lambda g(X, Y)=-(1+\alpha)(\xi f) g(\phi Y, X)+(2 k+\lambda) \eta(X) \eta(Y) .
$$

Interchanging $X$ and $Y$ in (5.8) we obtain

$$
S(Y, X)+\lambda g(Y, X)=-(1+\alpha)(\xi f) g(\phi X, Y)+(2 k+\lambda) \eta(Y) \eta(X)
$$

Adding (5.8) and (5.9) we get

$$
2 S(X, Y)+2 \lambda g(X, Y)=2(2 k+\lambda) \eta(X) \eta(Y)
$$

Using (5.10) in (5.1), we have

$$
\nabla_{Y} D f=(2 k+\lambda) \eta(Y) \xi
$$

Using (5.11) we compute $R(X, Y) D f$ and obtain

$$
g(R(X, Y)(\xi f) \xi, \xi)=2(2 k+\lambda) d \eta(X, Y)
$$

Thus we get

$$
2 k+\lambda=0
$$

Therefore from equation (5.7) we have $Y(\xi f)=0$, that is, $\xi f=c$, where $c$ is a constant. Thus the equation (5.5) gives $d f=c \eta$. Its exterior derivative implies that $c d \eta=0$, which implies $c=0$. Hence $f$ is a constant. Consequently (5.1) reduces to $S(X, Y)=2 k g(X, Y)$. Hence $M$ is Einstein. So we have the following:

Theorem 5.1. If the metric g of a three dimensional $N(k)$-contact metric manifold is a gradient Ricci soliton, then the manifold is an Einstein manifold. 
Since a 3-dimensional Einstein manifold is a manifold of constant curvature, hence we get the following:

Corollary 5.1. If the metric $g$ of a three dimensional $N(k)$-contact metric manifold is a gradient Ricci soliton, then the manifold is a manifold of constant curvature.

\section{Example of A $N(k)$-contact metric manifold}

We consider 3-dimensional manifold $M=\left\{(x, y, z) \in \mathbb{R}^{3},(x, y, z) \neq(0,0,0)\right\}$, where $(x, y, z)$ are the standard coordinate in $\mathbb{R}^{3}$. Let $e_{1}, e_{2}, e_{3}$ are three linearly independent vector fields in $\mathbb{R}^{3}$ which satisfies

$$
\left[e_{1}, e_{2}\right]=(1+\alpha) e_{3}, \quad\left[e_{2}, e_{3}\right]=2 e_{1} \quad \text { and } \quad\left[e_{3}, e_{1}\right]=(1-\alpha) e_{2},
$$

where $\alpha \neq \pm 1$ is a real number.

Let $g$ be the Riemannian metric defined by

$$
g\left(e_{1}, e_{3}\right)=g\left(e_{2}, e_{3}\right)=g\left(e_{1}, e_{2}\right)=0, \quad g\left(e_{1}, e_{1}\right)=g\left(e_{2}, e_{2}\right)=g\left(e_{3}, e_{3}\right)=1
$$

Let $\eta$ be the 1-form defined by

$$
\eta(U)=g\left(U, e_{1}\right)
$$

for any $U \in \chi(M)$. Let $\phi$ be the $(1,1)$-tensor field defined by

$$
\phi e_{1}=0, \phi e_{2}=e_{3}, \phi e_{3}=-e_{2}
$$

Using the linearity of $\phi$ and $g$ we have

$$
\begin{aligned}
\eta\left(e_{1}\right) & =1 \\
\phi^{2}(U) & =-U+\eta(U) e_{1}
\end{aligned}
$$

and

$$
g(\phi U, \phi W)=g(U, W)-\eta(U) \eta(W)
$$

for any $U, W \in \chi(M)$. Moreover

$$
h e_{1}=0, \quad h e_{2}=\alpha e_{2} \text { and } h e_{3}=-\alpha e_{3} .
$$

The Riemannian connection $\nabla$ of the metric tensor $g$ is given by Koszul's formula which is given by,

$$
2 g\left(\nabla_{X} Y, Z\right)=X g(Y, Z)+Y g(Z, X)-Z g(X, Y)
$$




$$
-g(X,[Y, Z])-g(Y,[X, Z])+g(Z,[X, Y])
$$

Using Koszul's formula we get the following:

$$
\begin{array}{lll}
\nabla_{e_{1}} e_{1}=0, & \nabla_{e_{1}} e_{2}=0, & \nabla_{e_{1}} e_{3}=0, \\
\nabla_{e_{2}} e_{1}=-(1+\alpha) e_{3}, & \nabla_{e_{2}} e_{2}=0, & \nabla_{e_{2}} e_{3}=(1+\alpha) e_{1}, \\
\nabla_{e_{3}} e_{1}=(1-\alpha) e_{2}, & \nabla_{e_{3}} e_{2}=-(1-\alpha) e_{1}, & \nabla_{e_{3}} e_{3}=0 .
\end{array}
$$

In view of the above relations we have

$$
\nabla_{X} \xi=-\phi X-\phi h X, \quad \text { for } \quad e_{1}=\xi
$$

Therefore the manifold is a contact metric manifold with the contact structure $(\phi, \xi, \eta, g)$.

Now, we find the curvature tensors as follows:

$$
\begin{array}{ll}
R\left(e_{1}, e_{2}\right) e_{2}=\left(1-\alpha^{2}\right) e_{1}, & R\left(e_{3}, e_{2}\right) e_{2}=-\left(1-\alpha^{2}\right) e_{3}, \\
R\left(e_{1}, e_{3}\right) e_{3}=\left(1-\alpha^{2}\right) e_{1}, & R\left(e_{2}, e_{3}\right) e_{3}=-\left(1-\alpha^{2}\right) e_{2}, \\
R\left(e_{2}, e_{3}\right) e_{1}=0, & R\left(e_{1}, e_{2}\right) e_{1}=-\left(1-\alpha^{2}\right) e_{2},
\end{array}
$$

In view of the expressions of the curvature tensors we conclude that the manifold is a $N\left(1-\alpha^{2}\right)$-contact metric manifold.

\section{Acknowledgement}

The authors are thankful to the referee for his/her valuable suggestions and comments towards the improvement of the paper.

\section{References}

[1] C. L. Bejan and M. Crasmareanu, Ricci solitons in manifolds with quasi-constant curvature, Publ. Math. Debrecen, 78(1) (2011), 235-243.

[2] D. E. Blair, Contact manifolds in Riemannian geometry, Lecture note in Math., 509, Springer-Verlag, BerlinNew York, 1976.

[3] D. E. Blair, T. Koufogiorgos and R. Sharma, A classification of 3-dimensional contact metric manifolds with $Q \phi=\phi Q$, Kodai Math. J., 13 (1990), 391-401.

[4] D. E. Blair, J. S. Kim and M. M. Tripathi, On the concircular curvature tensor of a contact metric manifold, J. Korean Math. Soc., 425 (2005), 883-992.

[5] E. Boeckx, O. Kowalski and L. Vanhecke, Riemannian manifolds of conullity two, Singapore World Sci. Publishing, 1996.

[6] E. Boeckx, A full classification of contact metric $(k, \mu)$-spaces, Illinois J. Math., 44 (2010), $212-219$.

[7] E. Cartan, Sur une classe remarqable d' espaces de Riemannian, Bull. Soc. Math. France., 54 (1962), $214-264$.

[8] U. C. De, C. Murathan and K. Arsalan, On the Weyl projective curvature tensor of an $N(k)$-contact metric manifold, Mathematica Panonoica, 21(1) (2010), 129-142. 
[9] U. C. De and A. Sarkar, On the quasi-conformal curvature tensor of a $(k, \mu)$-contact metric manifold, Math. Reports, 14(64), 2 (2012), 115-129.

[10] U. C. De, A. Yildiz and S. Ghosh, On a class of $N(k)$-contact metric manifolds, Math. Reports., 16(66)(2014), No. 2.

[11] Avik De and J. B. Jun, On $N(k)$-contact metric manifolds satisfying certain curvature conditions, Kyungpook Math. J., 51, 4 (2011), 457-468.

[12] J. B. Jun and U. K. Kim, On 3-dimensional almost contact metric manifolds, Kyungpook Math. J., 34(2) (1994), 293-301.

[13] O. Kowalski, An explicit classification of 3 - dimensional Riemannian spaces satisfying $R(X, Y) . R=0$, Czechoslovak Math. J., 46, 121 (1996), 427-474.

[14] C. Calin and M. Crasmareanu, From the Eisenhart problem to Ricci solitons in f-Kenmotsu manifolds, Bull. Malays. Math. Soc., 33(3) (2010), 361-368.

[15] J. T. Cho, Notes on contact Ricci solitons, Proc. Edinb. Math. Soc., 54(2011), 47-53.

[16] T. Chave and G. Valent, Quasi-Einstein metrics and their renoirmalizability properties, Helv. Phys. Acta., 69(1996), 344-347.

[17] T. Chave and G. Valent, On a class of compact and non-compact quasi-Einstein metrics and their renoirmalizability properties, Nuclear Phys. B., 478(1996), 758-778.

[18] B. Y. Chen, Some results on concircular vector fields and their applications to Ricci solitons, Bull. Korean Math. Soc., 52 (2015), 1535-1547.

[19] B. Y. Chen and S. Deshmukh, Ricci solitons and concurrent vector fields, Balkan J. Geom. Appl., 20 (2015), $14-25$.

[20] B. Y. Chen and S. Deshmukh, Classification of Ricci solitons on Euclidean hypersurfaces, Internat. J. Math., 25 (2014), no. 11, 1450104, 22 pp.

[21] Chen, B. Y. and Deshmukh, S., Geometry of compact shrinking Ricci solitons ., Balkan J. Geom. Appl. 19 (2014), no. 1, 13-21.

[22] B. Chow and D. Knoff, The Ricci flow: An introduction, Mathematical surveys and Monographs 110, American Math. Soc., 2004.

[23] U. C. De and S. Biswas, A note on $\xi$-conformally flat contact manifolds, Bull. Malays. Math. Soc., 29 (2006), 51-57.

[24] U. C. De and A. K. Mondal, Three dimensional Quasi-Sasakian manifolds and Ricci solitons, SUT J. Math., 48(1)(2012), 71-81.

[25] U. C. De and P. Majhi, On a type of contact metric manifolds, Lobacheviskii J. Math., 34(2013), 89-98.

[26] A. Derdzinski, Compact Ricci solitons, Preprint.

[27] A. Derdzinski, A Myers-type theorem and compact Ricci solitons, Proc. Amer. Math. Soc., 134(12)(2006), 36453648.

[28] S. Deshmukh, Jacobi-type vector fields on Ricci solitons, Bull. Math. Soc. Sci. Math. Roumanie, 55 (103), 1 (2012), 41-50.

[29] S. Deshmukh, H. Alodan and H. Al-Sodais, A Note on Ricci Soliton, Balkan J. Geom. Appl., 16(1) (2011), 48-55.

[30] D. Friedan, Non linera models in $2+\epsilon$ dimensions, Ann. Phys., 163(1985), 318-419.

[31] R. S. Hamilton, The Ricci flow on surfaces, Mathematics and general relativity (Santa Cruz, CA, 1986), $237-262$. Contemp. Math., 71, American Math. Soc., 1988.

[32] A. Ghosh, R. Sharma and J. T. Cho, Contact metric manifolds with $\eta$-parallel torsion tensor, Ann. Global Anal. Geom., 34 (3), 287-299.

[33] T. Ivey, Ricci solitons on compact 3-manifolds, Diff. Geom. Appl., 3 (1993), 301-307.

[34] T. Ivey, New examples of complete Ricci solitons, Proc. Amer. Math. Soc., 122(1) (1994), 241-245.

[35] P. Majhi and U. C. De, Classifications of $N(k)$-contact metric manifolds satisfying certain curvature conditions, Acta Math. Univ. Commenianae, LXXXIV (1)(2015), 167-178.

[36] C. Özgür, Contact metric manifolds with cyclic-parallel Ricci tensor, Diff. Geom. Dynamical Systems, 4 (2002), 21-25. 
[37] C. Özgür and S. Sular, On $N(k)$-contact metric manifolds satisfying certain conditions, SUT J. Math., 44(1)(2008), 89-99.

[38] G. Perelman, The entropy formula for the Ricci flow and its geometric applications, Preprint, http://arXiv.org/abs/Math.DG/0211159.

[39] Z. I.Szabó, Structure theorems on Riemannian spaces satisfying $R(X, Y) . R=0$, the local version, J. Diff. Geom., 17 (1982), 531-582.

[40] R. Sharma, Certain results on $K$-contact and ( $k, \mu$ )-contact manifolds, J. of Geometry, 89 (2008), $138-147$.

[41] L. Verstraelen, Comments on pseudosymmetry in the sense of Ryszard Deszcz, In: Geometry and Topology of submanifolds, VI. River Edge, NJ: World Sci. Publishing, 1994, 199-209.

[42] A. Taleshian and A. A. Hosseinzabeh, Investigation of Some Conditions on N(k)-Quasi Einstein Manifolds, Bull. Malays. Math. Soc., 34(3) (2011), 455-464.

[43] K. Yano and S. Bochner, Curvature and Betti numbers, Annals of mathematics studies, 32, Princeton university press, 1953.

[44] K. Yano, Concircular geometry I. concircular transformations, Proc. Imp. Acad. Tokyo, 16 (1940), $195-200$.

[45] Tanno, S., Ricci curvature of contact Riemannian manifolds, Tohoku Math. J., 40 (1988), 441-448.

[46] W. Wylie, Complete shrinking Ricci solitons have finite fundamental group, Proc. Amer. Math. Soc., 136(5) (2008), 1803-1806.

Department of Pure Mathematics, University of Calcutta, 35, Ballygaunge Circular Road, Kolkata -700019, West Bengal, India.

E-mail: uc_de@yahoo.com 